\title{
miR-27a suppresses the clonogenic growth and migration of human glioblastoma multiforme cells by targeting BTG2
}

\author{
WEI-QING LI ${ }^{1 *}$, HONG-YU YU ${ }^{1 *}$, NAN-ZHE ZHONG ${ }^{2 *}$, LI-JUN HOU ${ }^{2}$, YI-MING LI $^{2}$, \\ JIN HE ${ }^{1}$, HUI-MIN LIU ${ }^{1}$, CHUN-YAN XIA ${ }^{1}$ and YI-CHENG LU ${ }^{2}$ \\ Departments of ${ }^{1}$ Pathology and ${ }^{2}$ Neurosurgery, Changzheng Hospital, \\ Second Military Medical University, Shanghai 200003, P.R. China
}

Received October 2, 2014; Accepted November 28, 2014

DOI: $10.3892 /$ ijo.2015.2843

\begin{abstract}
R-27a and BTG2 are implicated in gliomagenesis and glioma progression. However, hitherto, a link between miR-27a and BTG2 in glioma has not been reported. In the present study, we investigated the effects of miR-27a on the proliferation and invasiveness of glioblastoma cells in vitro and in a mouse xenograft model and further studied the relation between miR-27a expression and its target gene BTG2, which was identified by computation prediction algorithms. Our MTT and clonogenic assays showed that miR-27a overexpression significantly increased the clonogenic growth of glioblastoma U87MG and U251MG cells. The Transwell assays further revealed that miR-27a overexpression markedly increased the number of migrated U87MG and U251MG cells. TargetScan and other prediction algorithms identified BTG2 as a target gene of miR-27a, which was confirmed by EGFP reporter and immunoblotting assays showing an inverse relation between miR-27a expression and endogenous BTG2 expression. BTG2 overexpression also increased the proliferation and invasiveness of glioblastoma cells and BTG2 functioned downstream of miR-27a in modulating the proliferation and migration of glioblastoma cells. In conclusion, miR-27a modulates human glioblastoma growth and invasion by targeting BTG2.
\end{abstract}

\section{Introduction}

Glioblastoma multiforme (GBM) is the most malignant form of human astrocytomas (1). Despite recent advances in our understanding of the molecular mechanisms of the disease

Correspondence to: Dr Yi-Ming Li or Dr Yi-Cheng Lu, Department of Neurosurgery, Changzheng Hospital, Second Military Medical University, Shanghai 200003, P.R. China

E-mail: yiyimig@163.com

E-mail: luyicheng10@gmail.com

*Contributed equally

Key words: glioblastoma multiforme, miR-27a, BTG2, proliferation, migration and improvement in therapeutic strategies, the median survival of GBM patients still remains dismal, with less than one year after diagnosis (2). GBM patients succumb to death due to rapid, aggressive and infiltrative growth of the tumor, rendering the tumor unresectable. Moreover, GBM is recalcitrant to radiotherapy and chemotherapy $(3,4)$ and patients eventually succumb to GBM despite the best available therapeutic regimen due to the emergence of chemoresistance to anticancer drugs.

MicroRNAs (miRNAs) are evolutionarily conserved, small non-coding endogenous RNA molecules that function as posttranscriptional gene regulators by pairing with the 3' untranslated regions (3'-UTR) of specific target messenger RNAs (mRNAs) to suppress translation or induce mRNA degradation $(5,6)$. Emerging evidence has revealed that many miRNAs function as oncogenes or anti-oncogenes and may play key roles in GBM initiation by regulating a myriad of target genes, especially PTEN, TP53 and EGFR, which are key regulators of GBM progression (7-10). miR-27a is located at chromosome 19 and has been shown to be expressed in a variety of cancer types including breast cancer, gastric adenocarcinoma and cervical cancer (11). miR-27a was shown to be an oncomir in prostate cancer by targeting prohibin (12). It was upregulated in breast cancer tissues (13) and has recently been proposed as a novel marker of breast cancer progression and worse prognosis (14). miR-27a was also found to be upregulated in glioma tissues (15) and may target multiple cellular signaling pathways important for gliomagenesis including the MAPK signaling pathway, the apoptotic signaling pathway and the transforming growth factor- $\beta$ (TGF- $\beta$ ) signaling pathway (16). miR-27a was shown to drive glioma cell growth by targeting the tumor suppressor gene MXI1 (17) and targeting miR-27a by anti-miRNAs suppressed the growth and invasiveness of GBM U87MG cells (18). These findings implicate miR-27a in gliomagenesis and progression.

The B-cell translocation gene 2 (BTG2), a p53-inducible anti-proliferation gene and a tumor suppressor gene (19), regulates the proliferation of neural progenitor cells during neurogenesis and promotes neurogenic asymmetric divisions (20). In a mouse model of platelet-derived growth factor B (PDGF-B) overexpression-induced glioma, Calzolar et al found that BTG2 downregulation was implicated in the process of malignant transformation and progression of low 
to high grade gliomas (21). Appolloni et al has further demonstrated that reduced BTG2 expression in human glioma tissues correlates with a significantly worse prognosis (22).

Given the important role of miR-27a in oncogenesis and progression of multiple cancer types, particularly glioma, we were interested in identifying downstream target genes of miR-27a that mediated the actions of miR-27a in GBM cells. In the present study, we investigated the effects of miR-27a on the proliferation and invasiveness of GBM cells in vitro and in a mouse xenograft model and further studied the relation between miR-27a expression and its target gene BTG2, which was identified by computation prediction algorithms.

\section{Materials and methods}

Animals. Eighteen female BALB/c-nu mice, 7-8 weeks of age, were obtained from the Institute of Zoology, Chinese Academy of Sciences, Shanghai, China and kept under specific pathogen-free conditions in accordance with procedures and guidelines set by the Institutional Animal Care and Use Committee (IACUC) of the Second Military Medical University, Shanghai, China. The study protocol was approved by the local institutional review board.

Plasmid construction. We amplified a DNA fragment containing the pri-miR-27a gene by PCR using the primer pair: 5'-ACG GGA TCC ACA TTG CCA GGG ATT TCC A-3' (sense) and 5'-CGC GAA TTC AGC TCA GTA GGC ACG GGA G-3' (antisense). The fragments were then inserted between $\mathrm{BamHI}$ and EcoRI sites of pcDNA3 (Invitrogen, Carlsbad, CA, USA), yielding pcDNA3/pri-miR-27a. For construction of the enhanced green fluorescence protein (EGFP) expression vector carrying the 3'-UTR segment of the BTG2, pcDNA3/ EGFP-BTG2 3'-UTR, we amplified the 3'-UTR segment of the $B T G 2$ gene carrying the predicted miR-27a binding site by PCR using the primer set: 5'-GAT CCA GAT GTG AGA GGG AGC AAG CAA GGT TAG CAA CTG TGA ACA GAG AGG TCG GGA TTT GCC CTG-3' (sense) and 5'-AAT TCA GGG CAA ATC CCG ACC TCT CTG TTC ACA GTT GCT AAC CTT GCT TGC TCC CTC TCA CAT CTG-3' (antisense). The PCR products were then cloned into the pcDNA3/EGFP vector between BamHI and EcoRI sites. To construct the siR-BTG2 vector, we performed an annealing reaction using two single strands 5'-CAC UAC AAA CAC CAC UGG UdTdT-3' and 5'-ACC AGT GGT GTT TGT AGT GdTdT-3'. To construct a BTG2 overexpression plasmid pcDNA3/BTG2 the full length human BTG2 gene (without the 3'-UTR) was PCR-amplified using a cDNA clone (GenBank ${ }^{\mathrm{TM}}$, AF361937) as a template with the primer set: 5'-ACG GGA TCC ATG AGC CAC GGG AAG GGA A-3' (sense) and CGC GAA TTC TAG CTG GAG ACT GCC ATC-3' (antisense), followed by insertion into the pcDNA3 vector between the EcoRI and XhoI sites. All insertions were verified by DNA sequencing.

Cells and transfection. The human GBM cell lines U87MG and U251MG (Cell Bank, Type Culture Collection, Chinese Academy of Sciences, Shanghai, China) were cultured in Dulbecco's modified Eagle's medium (DMEM) supplemented with $10 \%$ fetal bovine serum (FBS) and $100 \mathrm{U} / \mathrm{ml}$ penicillin/ streptomycin at $37^{\circ} \mathrm{C}$ in a humidified atmosphere with $5 \% \mathrm{CO}_{2}$.
To generate GBM cells expressing miR-27a or BTG2, we transfected the cells with pcDNA3/pri-miR-27a,pcDNA3/BTG2, or the control vector pcDNA3/EGFP using Lipofectamine 2000 (Invitrogen) according to the manufacturer's instructions. GBM cells with downregulated expression of BTG2 were also transfected with Lipofectamine 2000. Stable cell lines with downregulated expression of miR-27a were established by transfecting GBM cells with 2'-O-methyl-modified antisense oligonucleotide (ASO-miR-27a, Saierbio, Tianjin, China) followed by selection for 20-30 days in complete medium supplemented with $500 \mu \mathrm{g} / \mathrm{ml} \mathrm{G} 418$ (Invitrogen). Control cells were transfected with ASO-NC using the same procedure.

$R T$-PCR. Total cellular RNAs were isolated with the mirVana ${ }^{\mathrm{TM}}$ miRNA Isolation kit (Ambion, Austin, TX, USA) according to the manufacturer's protocol. For miRNA quantification, $1 \mu \mathrm{g}$ RNA was used as the template and cDNA was generated with the stem-loop reverse transcript primer and $M-M L V$ reverse transcriptase (Promega, Madison, WI, USA), followed by quantitative polymerase chain reaction (PCR) with SYBR Premix EX Taq (Takara, Otsu, Shiga, Japan) on an iQ5 real-time PCR detection system (Bio-Rad). To quantify the target gene BTG2, $2 \mu \mathrm{g}$ RNA was reverse-transcribed to cDNA with oligo(dT) primers and $M-M L V$ reverse transcriptase (Promega). The sequences of the primers used for the RT-PCR are provided in Table I. Raw data analysis was performed with iQ5 real-time PCR software. The relative fold-change in the transcripts was calculated using the $2^{-\Delta \Delta \mathrm{Ct}}$ method with U6 as a normalizing internal control for miRNAs and $\beta$-actin for BTG2. Each experiment was carried out in triplicate at least three times independently.

MTT and clonogenic assays. Logarithmically growing cells were plated into 96 -well plates at $2 \times 10^{3}$ cells/well. Cellular proliferation was evaluated using the 3-(4,5-dimethylthiazol2-yl)-2,5-diphenyltetrazolium bromide (MTT) assay as instructed by the manufacturer (Sigma-Aldrich, St. Louis, MO, USA). Absorbance was read at $570 \mathrm{~nm}$ using an uQuant Universal Microplate Spectrophotometer (Bio-Tek Instruments, Winooski, VT, USA). For clonogenic assays, GBM cells were seeded in 12 -well plates at $3 \times 10^{2}$ cells per well. The number of colonies was counted on the 9th day after seeding and a colony was counted only if it contained $>50$ cells. Each experiment was carried out in triplicate and at least three times independently.

Cell migration assays. Transwell cell migration assay was done to assess the migration of transfected GBM cells. Briefly, the GBM cells were seeded in Transwell inserts with $8.0-\mu \mathrm{m}$ pore Transwell filters (BD Biosciences, NJ, USA) in $0.2 \mathrm{ml}$ DMEM without FBS. The lower chamber was filled with $600 \mu \mathrm{l}$ DMEM containing $20 \%$ FBS acting as chemoattractants. Twentyeight hours later, the migrant cells that had attached to the lower surface were fixed with $20 \%$ methanol and stained for 20 min with crystal violet. The membranes were then carved and embedded under cover slips with the cells on the top. Cells in three different fields of view at x100 magnification were counted and expressed as the average number of cells per field of view. Each experiment was carried out in triplicate at least three times independently. 
Table I. Primer sequences for qRT-PCR.

\begin{tabular}{ll}
\hline Primers & \multicolumn{1}{c}{ Sequences (5'-3') } \\
\hline miR-27a-RT & GTCGTATCCAGTGCAGGGTCCGAGGTATTCG \\
& CACTGGATACGACGCGGAAC \\
U6-RT & GTCGTATCCAGTGCAGGGTCCGAGGTATTCG \\
& CACTGGATACGACAAAATATGGAAC \\
miR-27a-Fwd & TGCGGTTCACAGTGGCTAAG \\
U6-Fwd & TGCGGGTGCTCGCTTCGGCAGC \\
Reverse & CCAGTGCAGGGTCCGAGGT \\
$\beta$-actin-S & CGTGACATTAAGGAGAAGCTG \\
$\beta$-actin-A & CTAGAAGCATTTGCGGTGGAC \\
BTG2-qPCR-S & GAACTGTTGCGTGCTTGA \\
BTG2-qPCR-A & ACCTTGCTTGCTCCCTCT \\
\hline
\end{tabular}

Tumor xenografts. For GBM xenograft experiments, 5x10 stable U87MG cells with downregulated miR-27a were inoculated subcutaneously at the axillary fossae of female athymic nude mice. Tumor size was monitored by measuring the length and width with calipers and volumes were calculated with the formula: $\left(\mathrm{LxW}^{2}\right) \times 0.5 \mathrm{~mm}^{3}$, where $\mathrm{L}$ is the length and $\mathrm{W}$ is the width of each tumor.

Cell cycle analysis. Transfected GBM cells were plated in 6-well plates in duplicate for $24 \mathrm{~h}$ in complete medium. One group of cells was deprived of serum for $24 \mathrm{~h}$ before harvesting, while another group of cells was returned to complete medium for another $24 \mathrm{~h}$ before harvesting. Cells were gathered by centrifugation, fixed in $95 \%$ (v/v) ethanol and stored at $-20^{\circ} \mathrm{C}$ overnight. After washing with phosphatebuffered saline (PBS), the cells were resuspended in propidium iodide staining buffer (PBS, $0.1 \%$ Triton X-100, $60 \mu \mathrm{g} / \mathrm{ml}$ propidium iodide, $0.1 \mathrm{mg} / \mathrm{ml}$ DNase-free RNase and $0.1 \%$ trisodiumcitrate) for $30 \mathrm{~min}$ on ice. The DNA content was analyzed with FACSCalibur flow cytometer and CellQuest software (BD Biosciences). The proliferation index (PI) was calculated using the following equation:

$$
P I=(S+G 2) / G 1
$$

where $S, G 2$ and $G 1$ represent the percentage of cells in the $S$, $G 2$ and $G 1$ phase, respectively.

Computational algorithm analysis. We obtained predicted miRNA target sites of miR-27a on human mRNA transcripts from PicTar (http://picta.mdc-berlin.de/), TargetScan (www. targetscan.org/) (Whitehead Institute for Biomedical Research, Cambridge, MA, USA) and miRBase Targets databases (23).

Fluorescent reporter assays. For determination of direct interaction between miR-27a and BTG2 mRNA, 1.5x10 $487 \mathrm{MG}$ cells per well were seeded into 48 -well plates and after 24-h incubation, cells were transfected with pri-miR-27a or ASO-miR-27a together with the pcDNA3/EGFP-BTG2 3'-UTR reporter vector, the RFP expression vector pDsRed2-N1 (Clontech) was used as an internal control. Fortyeight hours after transfection, cellular lysates were prepared using RIPA lysis buffer (150 mM NaCl, $50 \mathrm{mM}$ Tris- $\mathrm{HCl}$ $\mathrm{pH} 8.0,1 \%$ Triton $\mathrm{X}-100$ and $0.1 \%$ SDS) and EGFP and RFP intensity was then measured with an F-4500 fluorescence spectrophotometer (Hitachi, Tokyo, Japan). All experiments were performed at least three times independently.

Western blot assays. Cellular lysates were prepared using RIPA lysis buffer as described above and subjected to $15 \%$ SDS denaturing polyacrylamide gel electrophoresis. The immunoblotting procedure was performed as previously described (24) and anti-BTG2 or anti-GAPDH antibodies (Abcam) were used for the procedure. The protein bands were visualized by enhanced chemiluminescence and analyzed using the LabWorks image acquisition and analysis software (UVP, LLC).

Statistical analysis. The data are reported as mean \pm SD of at least three independent experiments with the two-sided Student's t-test. A P-value of $<0.05$ was considered significant.

\section{Results}

miR-27a promotes the clonogenic growth and migration of U87MG cells. We were interested in the effect of miR-27a, which has been shown to be significantly overexpressed in GBM tissues (25), on the proliferation and migration of GBM cells. Our quantitative RT-PCR assays revealed that transfection with pri-miR-27a caused a significant 3.97 \pm 0.35 -fold increase in miR-27a levels compared with controls $(\mathrm{P}<0.001)$ in GBM U87MG and U251MG cells while transfection with ASO-miR-27a caused a marked $78 \pm 6 \%$ reduction in miR-27a levels in the two cell lines $(\mathrm{P}<0.01)$ (Fig. 1A). The MTT assays showed that miR-27a overexpression was associated with an $\sim 1.47 \pm 0.02$-fold increase in the viability of U87MG and U251MG cells over the controls ( $\mathrm{P}<0.05$ or 0.01 ) (Fig. 1B). A corresponding significant increase in the number of colonies of U87MG and U251MG cells was also observed as revealed by clonogenic assays $(\mathrm{P}<0.05$ or 0.01$)$ (Fig. 1C). By contrast, miR-27a downregulation noticeably suppressed the viability and clonogenicity of U87MG and U251MG cells $(\mathrm{P}<0.001$ or 0.01) (Fig. 1B and C). Transwell assays further revealed that miR-27a overexpression significantly increased the number of migrated U87MG and U251MG cells $(\mathrm{P}<0.05$ or 0.001$)$ while miR-27a downregulation by ASO-miR-27a caused a $50 \%$ reduction in the number of migrated cells $(\mathrm{P}<0.05$ or 0.001) (Fig. 1D). We also found that miR-27a downregulation markedly reduced the growth of mouse xenografts bearing U87MG cells ( $\mathrm{P}<0.05$ vs. controls) (Fig. 2). The above findings together indicated that miR-27a overexpression potentiates the growth and migration of GBM cells both in vitro and in vivo.

miR-27a facilitates G1/S phase transition in GBM cells. We determined whether increased proliferation of GMB cells by miR-27a overexpression was due to changes in cell cycle distribution. Our flow cytometric analysis showed that miR-27a overexpression by pri-miR-27a caused an apparent increase in the percentage of U87MG (pri-miR-27a, 27.94 $\pm 0.72 \%$ vs. controls, $21.65 \pm 0.39 \% \mathrm{P}<0.05$ ) and $\mathrm{U} 251 \mathrm{MG}$ cells (primiR-27a, 33.97 $\pm 1.63 \%$ vs. controls, $26.64 \pm 2.12 \% \mathrm{P}<0.05)$ in the $\mathrm{S}$ phase (Fig. 3A and B). By contrast, downregulation by 

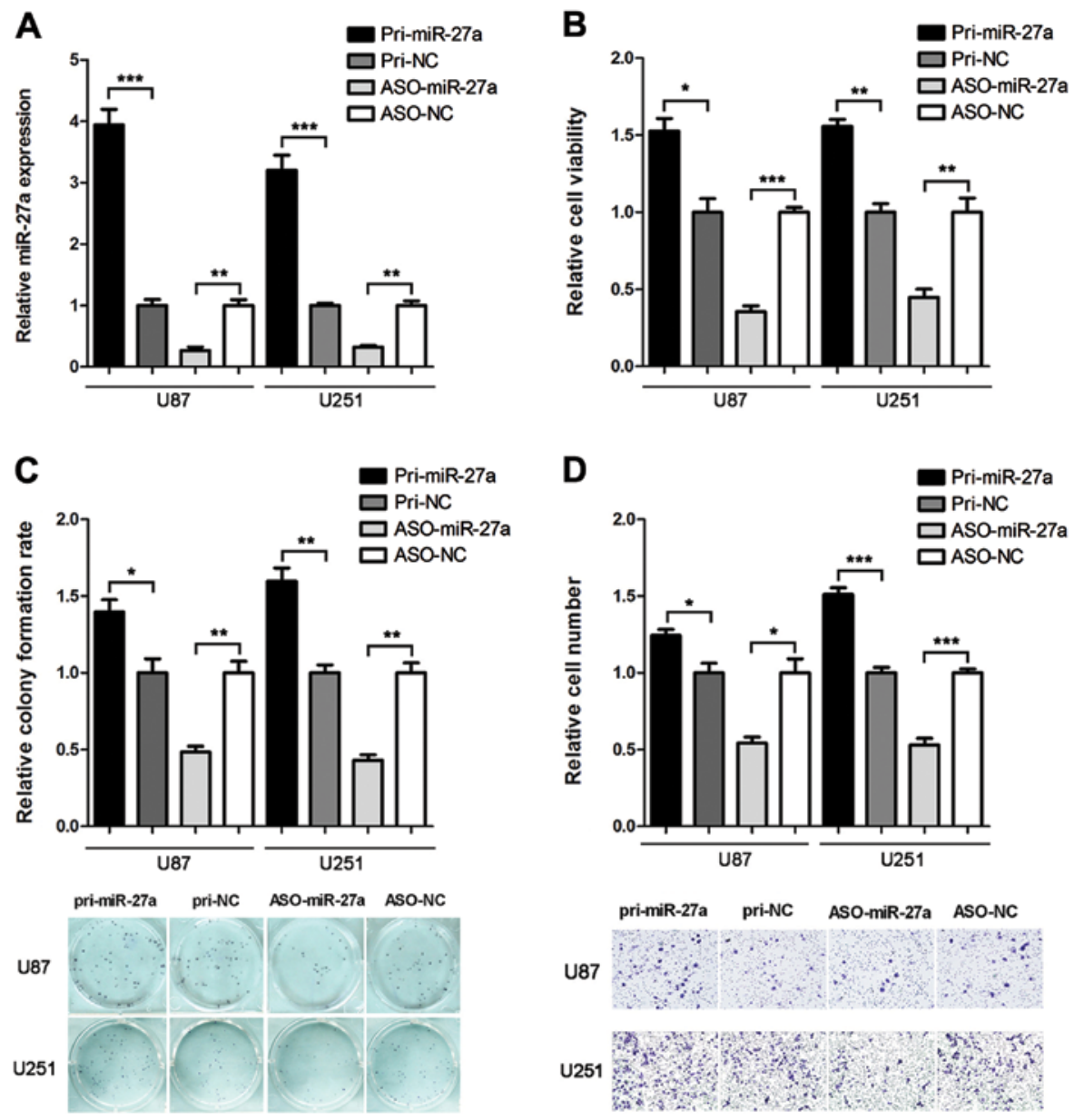

Figure 1. miR-27a promotes the proliferation and migration of glioblastoma cells. (A) U87MG and U251MG cells were transfected with pri-miR-27a or ASO-miR-27a as detailed in Materials and methods and miR-27a expression was examined by quantitative RT-PCR and normalized against U6 snRNA. (B) Cell viability was evaluated using the 3-(4,5-dimethylthiazol-2-yl)-2,5-diphenyltetrazolium bromide (MTT) assay. (C) The effect of miR-27a on clonogenicity of glioblastoma multiforme (GBM) cells was evaluated by colony formation assays as detailed in Materials and methods. (D) Migration of U87MG and U251MG cells was assessed by Transwell assays. Data are expressed as mean \pm SD of at least three independent experiments. (C and D) Representative images are shown in the lower panels. ${ }^{*} \mathrm{P}<0.05,{ }^{* *} \mathrm{P}<0.01$ and ${ }^{* * *} \mathrm{P}<0.001$.
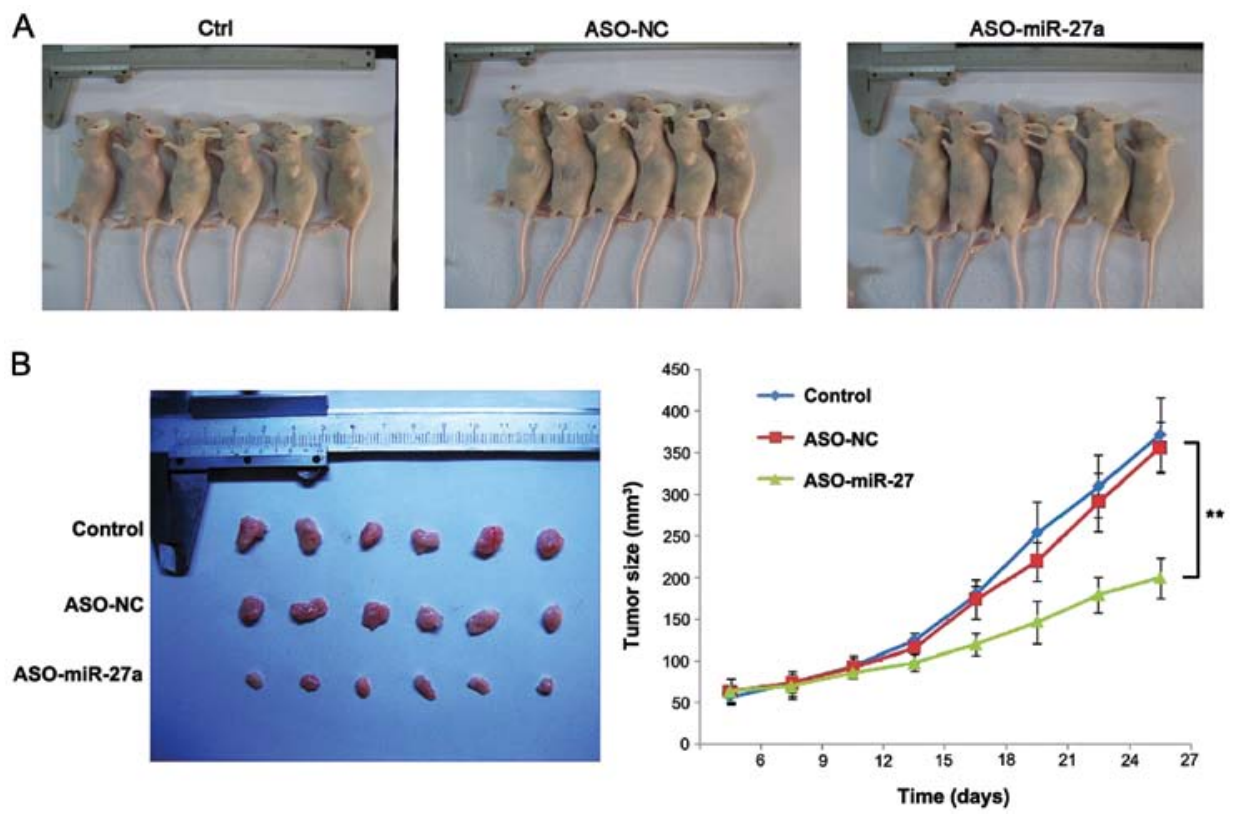

Figure 2. miR-27a promotes the proliferation and migration of glioblastoma cells. (A) The image of the nude mice subcutaneously injected with U87MG cells transfected with ASO-miR-27a, ASO-NC and the control group (n=6). (B) Mouse xenografts bearing stable U87MG cells with downregulated miR-27a were established as described in Materials and methods. Tumor growth was evaluated by measuring tumor volume over time. Data are expressed as mean \pm SD of at least three independent experiments. ${ }^{*} \mathrm{P}<0.05,{ }^{* *} \mathrm{P}<0.01$ and ${ }^{* * * *} \mathrm{P}<0.001$. 
A
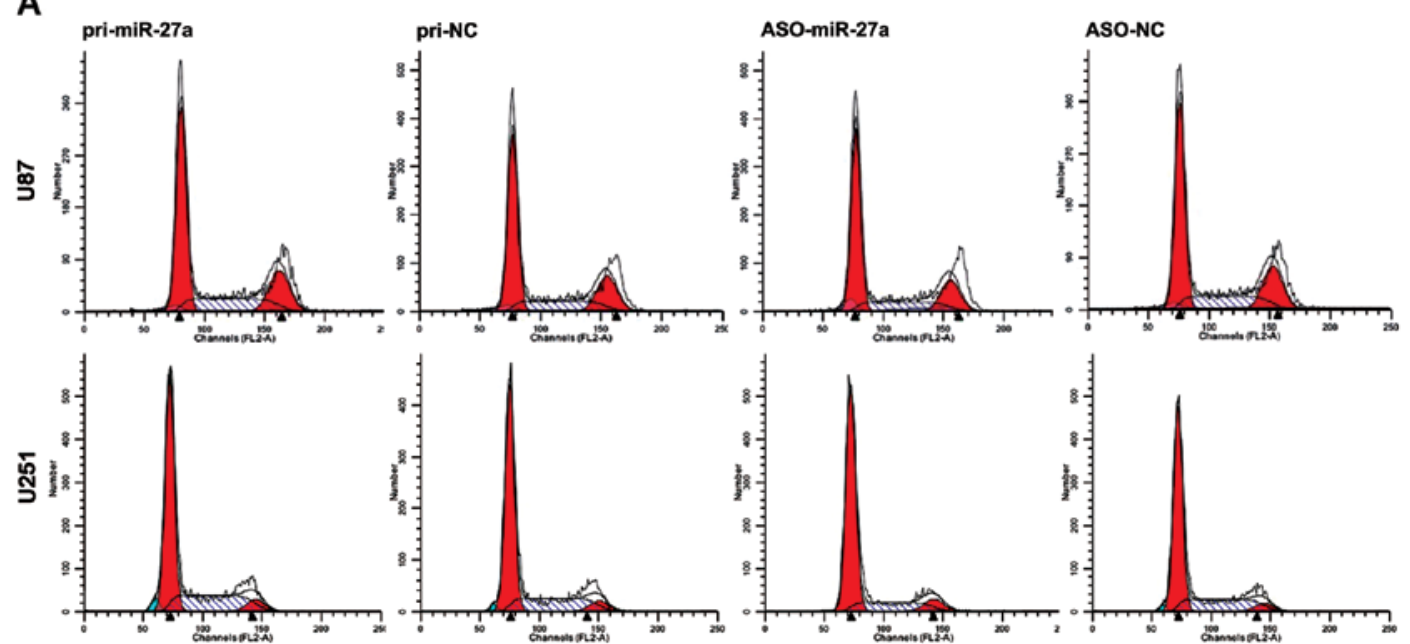

B
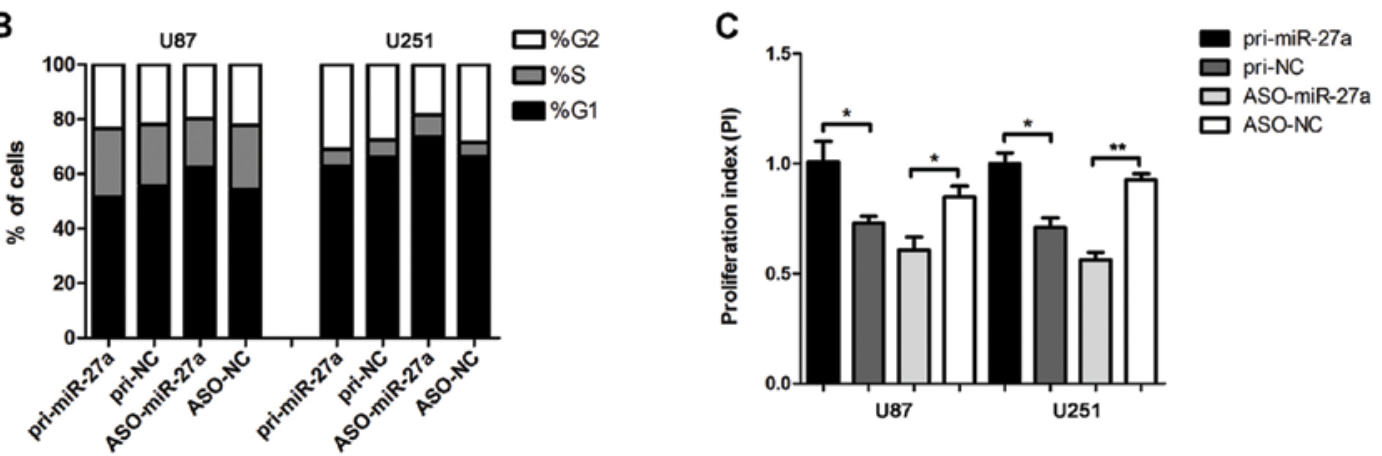

Figure 3. miR-27a facilitates G1/S phase transition in glioblastoma cells. GBM U87MG and U251MG cells were treated as described in Fig. 1 and cell cycle distribution was analyzed by flow cytometry. Representative histograms are shown (A) and the percentage of U87MG and U251MG cells in G1, S and G2 phase is shown (B). The proliferation index (PI) of treated U87MG and U251MG cells is shown (C). (B and C) Data are expressed as mean \pm SD of at least three independent experiments. ${ }^{*} \mathrm{P}<0.05$.

A

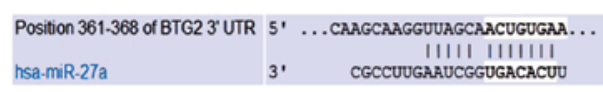

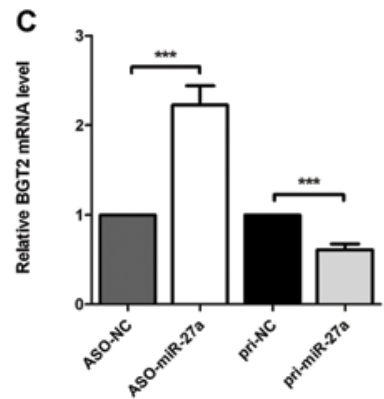

B
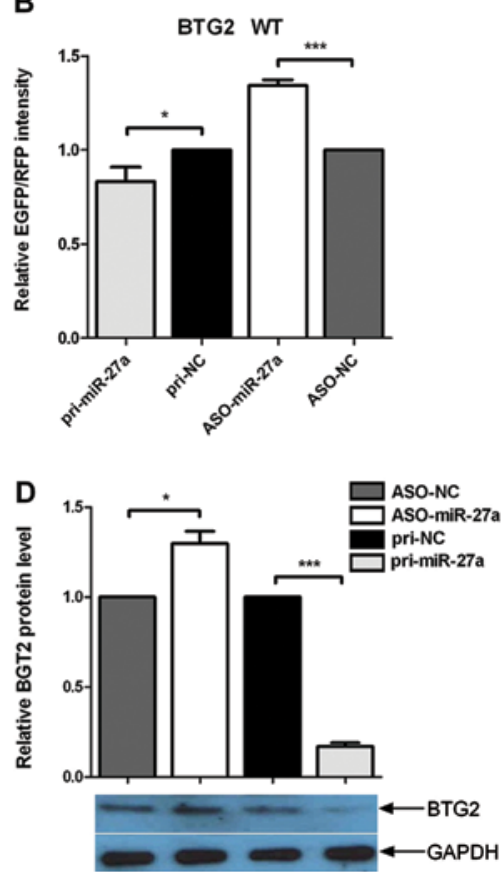

Figure 4. miR-27a negatively regulates the expression of BTG2 by directly targeting its 3'-UTR. (A) Computer algorithms TargetScan, PicTar and miRBase Targets reveal the presence of a potential binding site for miR-27a in the BTG2 3'-UTR. U87MG cells were co-transfected with pcDNA3/EGFP-BTG2 3'-UTR and pri-miR-27a or ASO-miR-27a as detailed in Materials and methods. EGFP reporter assays were done to examine binding of miR-27a to the BTG2 3'-UTR (B) and quantitative RT-PCR (C) and immunoblotting assays were done to examine effect of miR-27a overexpression or downregulation on BTG2 expression. The control was normalized to 1. (B-D) Data are expressed as mean \pm SD of at least three independent experiments. ${ }^{*} \mathrm{P}<0.05$ and ${ }^{* * *} \mathrm{P}<0.001$. 

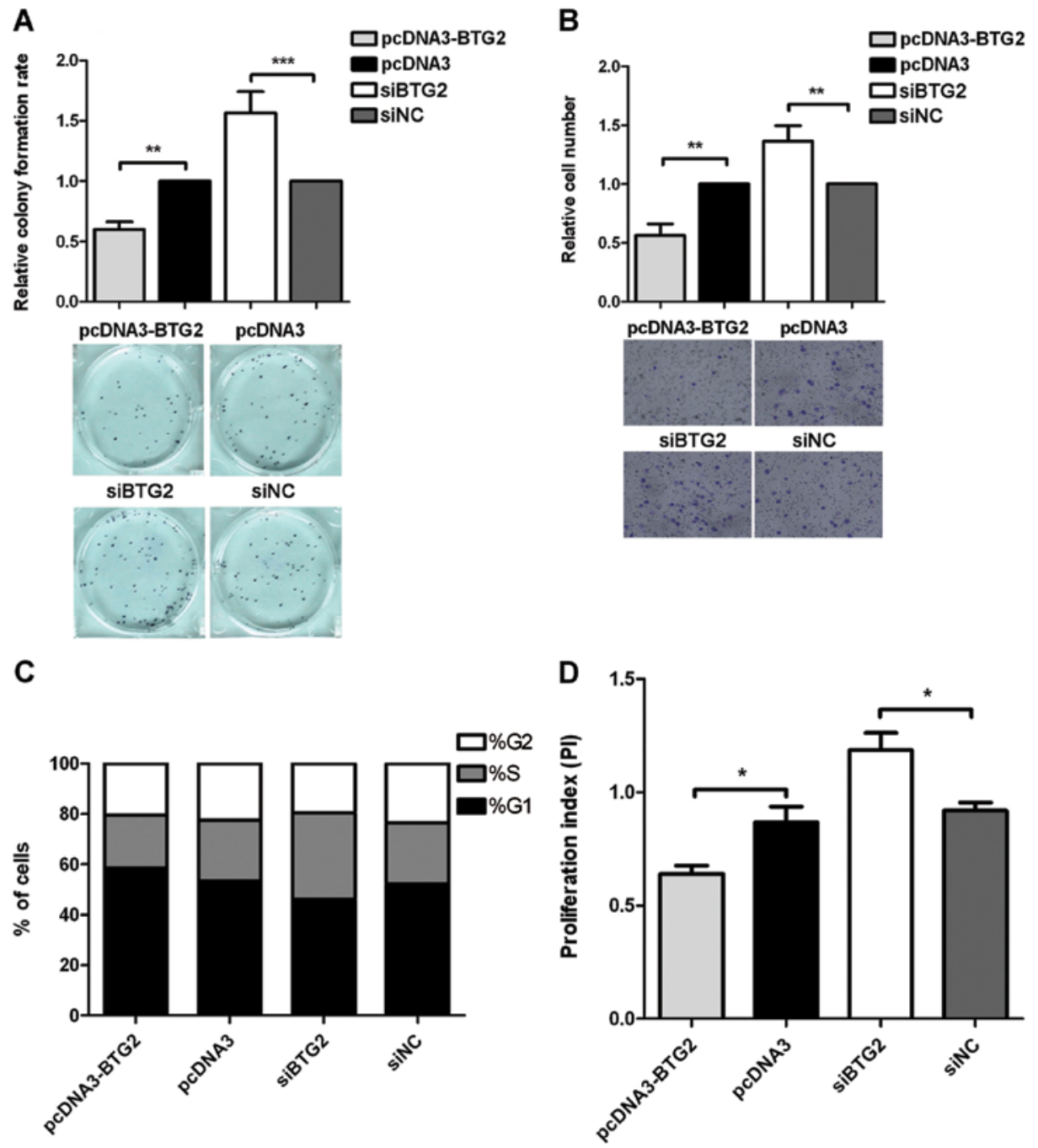

Figure 5. BTG2 modulates colony formation and migration of U87MG cells. (A) U87MG cells were transfected with pCDN3-BTG2 or small interfering RNA (siRNA) against BTG2 and appropriate controls as detailed in Materials and methods. Colony formation was evaluated by clonogenic assays as described in Materials and methods. (B) Migration of U87MG cells was examined by Transwell assays. The control was normalized to 1. (A and B) Representative images are shown in the lower panels. (C) Changes in cell cycle distributions of U87MG cells were determined by flow cytometry and the PI is calculated (D). Data are expressed as mean $\pm \mathrm{SD}$ of at least three independent experiments. ${ }^{*} \mathrm{P}<0.05,{ }^{* *} \mathrm{P}<0.01$ and ${ }^{* * *} \mathrm{P}<0.001$.

A

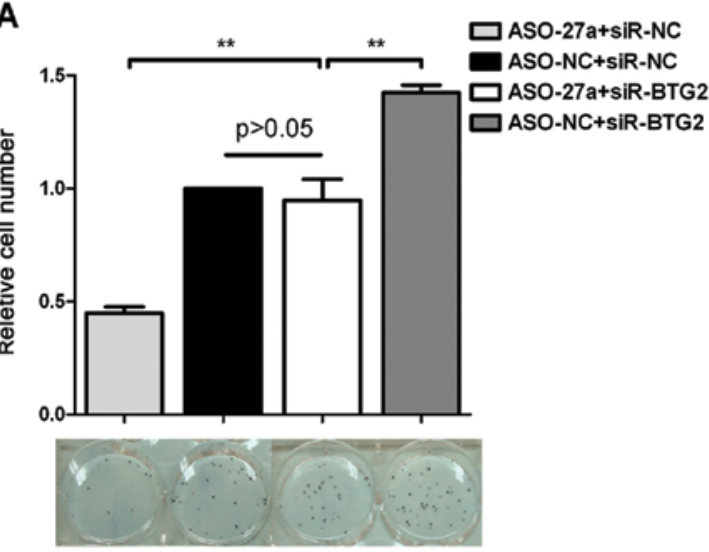

B

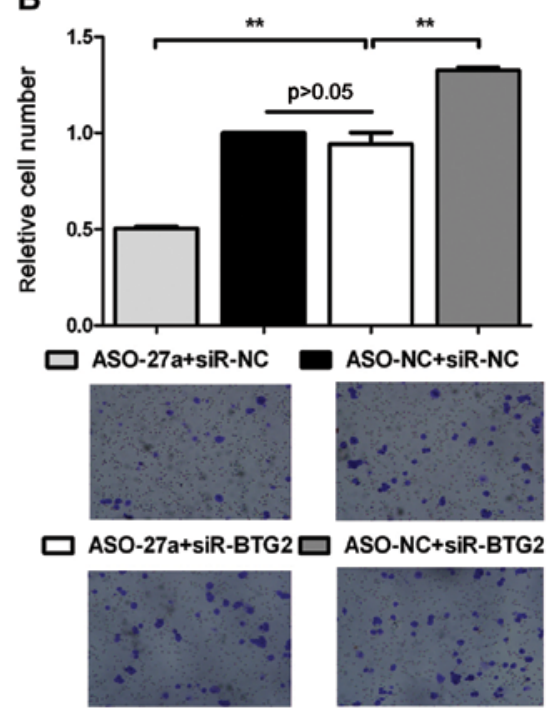

Figure 6. BTG2 functions downstream of miR-27a. (A) U87MG cells were transfected with ASO-miR-27a or siR-BTG2 and appropriate controls as detailed in Materials and methods and colony formation was evaluated using clonogenic assays (A) and migration of U87MG cells by Transwell assays (B). Data are expressed as mean $\pm \mathrm{SD}$ of at least three independent experiments. (A and B) Representative images are shown in the lower panels. ${ }^{* *} \mathrm{P}<0.01$. 
ASO-miR-27a noticeably decreased the percentage of U87MG (ASO-miR-27a, 15.87 \pm 0.95 vs. controls, 24.53 $\pm 0.24 \%$ $\mathrm{P}<0.05$ ) and U251MG cells (ASO-miR-27a, 17.58 \pm 1.39 vs. controls, $28.56 \pm 3.51 \% \mathrm{P}<0.05$ ) in the $\mathrm{S}$ phase (Fig. $3 \mathrm{~A}$ and $\mathrm{B}$ ). Consistently, U87MG and U251MG cells with miR-27a overexpression showed a significantly higher proliferation index than that of controls $(\mathrm{P}<0.05$ while GBM cells with downregulated miR-27a had a noticeably lower proliferation index compared with controls $(\mathrm{P}<0.01$ or 0.05$)$ (Fig. $3 \mathrm{C})$. These results suggest that miR-27a may promote the clonogenic growth of GBM cells by increasing the percentage of GBM cells in the $\mathrm{S}$ phase.

miR-27a negatively regulates the expression of BTG 2 by directly targeting its 3'-UTR. We used TargetScan, PicTar and miRBase targets to identify candidate target genes of miR-27a and found that the $B T G 2$ gene, whose encoded protein is involved in cell cycle control (26), contained a putative binding site for miR-27a in its 3'-UTR (Fig. 4A). To confirm whether BTG2 was a bona fide target of miR-27a, we co-transfected U87MG cells with pcDNA3/EGFP-BTG2 3'-UTR and pri-miR-27a or ASO-miR-27a. We found that pri-miR-27a caused a $19.7 \pm 2.4 \%$ reduction in EGFP intensity compared to controls $(\mathrm{P}<0.05)$ while ASO-miR-27a caused a $37.2 \pm 0.7 \%$ increase in EGFP intensity ( $\mathrm{P}<0.001$ vs. controls) (Fig. 4B). Our quantitative RT-PCR assays showed that miR-27a overexpression caused a marked $39.3 \pm 1.1 \%$ decrease while miR-27a downregulation by ASO-miR-27a caused a noticeable $108.6 \pm 9.6 \%$ increase in the mRNA transcript levels of BTG2 ( $<<0.001$ vs. controls in both) (Fig. 4C). Immunoblotting assays further revealed that miR-27a overexpression led to a marked $79.3 \pm 2.1 \%$ decrease $(\mathrm{P}<0.001$ vs. controls) while miR-27a downregulation caused a significant $22.7 \pm 4.4 \%$ increase ( $P<0.05$ vs. controls) in endogenous BTG2 levels (Fig. 4D). These data reveal that miR-27a negatively regulates endogenous BTG2 expression by targeting its 3'-UTR.

BTG2 modulates the growth and migration of GBM cells. We examined whether BTG2 exerted any effect on the clonogenic growth and migration of GBM cells. Our clonogenic assays demonstrated that suppression of BTG2 expression by siRNA caused a 1.6-fold increase in the number of colonies from U87MG cells ( $\mathrm{P}<0.001$ vs. controls) while BTG2 overexpression caused a $60 \%$ reduction in the number of colonies $(\mathrm{P}<0.01$ vs. controls) (Fig. 5A). Moreover, BTG2 knockdown was associated with 1.4-fold increase in the number of migrated U87MG cells ( $\mathrm{P}<0.01$ vs. controls) (Fig. 5B). By contrast, BTG2 overexpression was associated with a $55 \%$ decrease in the number of migrated U87MG cells ( $\mathrm{P}<0.01$ vs. controls). We further assessed the effect of BTG2 on cell cycle distributions by flow cytometry. We found that BTG2 knockdown markedly increased the proportion of cells in the $S$ phase $(\mathrm{P}<0.05$ vs. controls) while BTG2 overexpression led to a significant decline in the proportion of U87MG cells in the $\mathrm{S}$ phase $(\mathrm{P}<0.05$ vs. controls) (Fig. 5C). Consistently, BTG2 downregulation significantly increased while BTG2 overexpression markedly reduced the proliferation index $(\mathrm{P}<0.05$ in both) (Fig. 5D). These data suggest that BTG2, similar to miR-27a, modulated the clonogenic growth and migration of human GBM cells.
BTG2 functions downstream of miR-27a in modulating the proliferation and migration of human GBMcells. To determine whether the effects of miR-27a expression on cell growth and migration of GBM cells were mediated by BTG2, we transfected U87MG cells with ASO-miR-27a or siR-BTG2 and appropriate controls. Consistently, miR-27a downregulation by ASO-miR-27a markedly suppressed the clonogenic growth of U87MG cells ( $\mathrm{P}<0.01$ vs. controls); this decrease, however, was abrogated by siRNA knockdown of BTG2 (Fig. 6A). Transwell assays further showed that knockdown of BTG2 reversed the suppression of U87MG cell migration by ASO-miR-27a from 50-90\% of controls (Fig. 6B). These results indicate that BTG2 functions downstream of miR-27a in modulating the proliferation and migration of human GBM cells.

\section{Discussion}

miR-27a and BTG2 have been shown to be implicated in gliomagenesis and glioma progression. However, hitherto, a link between miR-27a and BTG2 in glioma has not been suspected. The present study demonstrated that miR-27a modulates the growth and invasiveness of GBM cells and directly targets BTG2.

We showed that miR-27a overexpression significantly increased the clonogenic growth of GBM cells in vitro. Furthermore, miR-27a overexpression was associated with markedly increased invasiveness of GBM cells in vitro. These findings are consistent with those by $\mathrm{Xu}$ et al (17) who found that miR-27a was upregulated in glioma tissues and its overexpression drove the proliferation of glioma cells. miR-27a has been reported to regulate cell growth and division in a dosedependent manner (11). We found that increased proliferation of GBM cells from miR-27a overexpression was associated with a marked increase in the proportion of GBM cells in the $\mathrm{S}$ phase and a corresponding increase in the proliferation index. We further showed that miR-27a downregulation caused a significant reduction in the number of migrated GBM cells as revealed by Transwell assays. This is in agreement with the findings by Feng et al who showed inhibition of miR-27a substantially impaired the invasiveness of U87MG cells in vitro (18).

BTG2 downregulation was involved in the progression of low to high grade gliomas (21) in a mouse glioma model and reduced BTG2 expression was found to correlate with a worse prognosis (22) of glioma patients, suggesting that BTG2 may play an important role in gliomagenesis and progression. Our computational prediction algorithms revealed BTG2 as a candidate target gene of miR-27a, which was confirmed by our findings from EGFP reporter assays showing an inverse relation between miR-27a expression and endogenous BTG2 expression. Though molecular targets of miR-27a such as Sprouty have been reported (27), no previous study has shown that miR-27a targets BTG2. We showed that BTG2 expression impacts on the proliferation and invasiveness of GBM cells and BTG2 functions downstream of miR-27a in modulating the proliferation and migration of GBM cells.

It has been reported that BTG2 is a direct transcriptional target gene of p53 (28-31). BTG2 was found to trigger cell cycle arrest by inhibiting G1-S transition via decreasing cyclin D1 
expression $(19,32,33)$. Apart from glioma (22), downregulation of BTG2 expression has also been observed in several tumor types, including prostate cancer, clear renal cell carcinomas and breast cancer (34-36). We speculate that miR-27a functions as an oncomir through negative regulation of BTG2, which then promotes $\mathrm{G} 1 \rightarrow \mathrm{S}$ transition.

Collectively, our results indicate that miR-27a and BTG2 promote the migration and the growth of GBM in vitro and in vivo and miR-27a directly targets BTG2 in GBM cells. Our findings suggest that miR-27a and BTG2 may represent novel biomarkers for GBM patients and could potentially be manipulated therapeutically.

\section{Acknowledgements}

This study was supported by grants from Shanghai Municipal Science and Technology Commission (no. 10ZR1439000 to Dr Yi-Ming Li), the National Natural Sciences Fund Project of China (NSFC no. 81101656/H1609 to Dr Yi-Ming Li, NSFC no. 81201987/H1618 to Dr Wei-Qing Li and NSFC no. 30930094 to Dr Yi-Cheng Lu).

\section{References}

1. Furnari FB, Fenton T, Bachoo RM, et al: Malignant astrocytic glioma: genetics, biology, and paths to treatment. Genes Dev 21: 2683-2710, 2007.

2. Robe PA, Martin DH, Nguyen-Khac MT, et al: Early termination of ISRCTN45828668, a phase $1 / 2$ prospective, randomized study of sulfasalazine for the treatment of progressing malignant gliomas in adults. BMC Cancer 9: 372, 2009.

3. Van Meir EG, Hadjipanayis CG, Norden AD, Shu HK, Wen PY and Olson JJ: Exciting new advances in neuro-oncology: the avenue to a cure for malignant glioma. CA Cancer J Clin 60: 166-193, 2010.

4. Stupp R, Mason WP, van den Bent MJ, et al: Radiotherapy plus concomitant and adjuvant temozolomide for glioblastoma. N Engl J Med 352: 987-996, 2005.

5. Bartel DP: MicroRNAs: target recognition and regulatory functions. Cell 136: 215-233, 2009.

6. Pillai RS: MicroRNA function: multiple mechanisms for a tiny RNA? RNA 11: 1753-1761, 2005.

7. Chan JA, Krichevsky AM and Kosik KS: MicroRNA-21 is an antiapoptotic factor in human glioblastoma cells. Cancer Res 65: 6029-6033, 2005.

8. Zhou X, Ren Y, Moore L, et al: Downregulation of miR-21 inhibits EGFR pathway and suppresses the growth of human glioblastoma cells independent of PTEN status. Lab Invest 90: 144-155, 2010.

9. Suh SS, Yoo JY, Nuovo GJ, et al: MicroRNAs/TP53 feedback circuitry in glioblastoma multiforme. Proc Natl Acad Sci USA 109: 5316-5321, 2012.

10. Rao SA, Arimappamagan A, Pandey P, et al: miR-219-5p inhibits receptor tyrosine kinase pathway by targeting EGFR in glioblastoma. PLoS One 8: e63164, 2013.

11. Liu T, Tang H, Lang Y, Liu M and Li X: MicroRNA-27a functions as an oncogene in gastric adenocarcinoma by targeting prohibitin. Cancer Lett 273: 233-242, 2009.

12. Fletcher CE, Dart DA, Sita-Lumsden A, Cheng H, Rennie PS and Bevan CL: Androgen-regulated processing of the oncomir miR-27a, which targets Prohibitin in prostate cancer. Hum Mol Genet 21: 3112-3127, 2012.

13. Gasparini P, Cascione L, Fassan M, et al: microRNA expression profiling identifies a four microRNA signature as a novel diagnostic and prognostic biomarker in triple negative breast cancers. Oncotarget 5: 1174-1184, 2014

14. Tang W, Zhu J, Su S, et al: MiR-27 as a prognostic marker for breast cancer progression and patient survival. PLoS One 7: e51702, 2012.

15. Skog J, Wurdinger T, van Rijn S, et al: Glioblastoma microvesicles transport RNA and proteins that promote tumour growth and provide diagnostic biomarkers. Nat Cell Biol 10: 1470-1476, 2008
16. Yang S, Wang K, Qian C, et al: A predicted miR-27a-mediated network identifies a signature of glioma. Oncol Rep 28: 1249-1256, 2012

17. Xu W, Liu M, Peng X, et al: miR-24-3p and miR-27a-3p promote cell proliferation in glioma cells via cooperative regulation of MXI1. Int J Oncol 42: 757-766, 2013.

18. Feng SY, Dong CG, Wu WK, Wang XJ, Qiao J and Shao JF: Lentiviral expression of anti-microRNAs targeting miR-27a inhibits proliferation and invasiveness of U87 glioma cells. Mol Med Rep 6: 275-281, 2012.

19. Kawakubo H, Carey JL, Brachtel E, et al: Expression of the NF-kappaB-responsive gene BTG2 is aberrantly regulated in breast cancer. Oncogene 23: 8310-8319, 2004.

20. Malatesta P, Gotz M, Barsacchi G, Price J, Zoncu R and Cremisi F: PC3 overexpression affects the pattern of cell division of rat cortical precursors. Mech Dev 90: 17-28, 2000.

21. Calzolari F, Appolloni I, Tutucci E, et al: Tumor progression and oncogene addiction in a PDGF-B-induced model of gliomagenesis. Neoplasia 10: 1373-1382, 2008.

22. Appolloni I, Curreli S, Caviglia S, et al: Role of Btg2 in the progression of a PDGF-induced oligodendroglioma Model. Int J Mol Sci 13: 14667-14678, 2012.

23. Griffiths-Jones S, Grocock RJ, van Dongen S, Bateman A and Enright AJ: miRBase: microRNA sequences, targets and gene nomenclature. Nucleic Acids Res 34: D140-D144, 2006.

24. Liang C, Guo S and Yang L: All-trans retinoic acid upregulates VEGF expression in glioma cells in vitro. J Biomed Res 27: $51-55,2013$

25. Ge YF, Sun J, Jin CJ, Cao BQ, Jiang ZF and Shao JF: AntagomiR-27a targets FOXO3a in glioblastoma and suppresses U87 cell growth in vitro and in vivo. Asian Pac J Cancer Prev 14: 963-968, 2013.

26. Donato LJ, Suh JH and Noy N: Suppression of mammary carcinoma cell growth by retinoic acid: the cell cycle control gene $\mathrm{Btg} 2$ is a direct target for retinoic acid receptor signaling. Cancer Res 67: 609-615, 2007.

27. Ma Y, Yu S, Zhao W, Lu Z and Chen J: miR-27a regulates the growth, colony formation and migration of pancreatic cancer cells by targeting Sprouty2. Cancer Lett 298: 150-158, 2010.

28. Kannan K, Kaminski N, Rechavi G, Jakob-Hirsch J, Amariglio N and Givol D: DNA microarray analysis of genes involved in p53 mediated apoptosis: activation of Apaf-1. Oncogene 20: 3449-3455, 2001.

29. Boiko AD, Porteous S, Razorenova OV, Krivokrysenko VI, Williams BR and Gudkov AV: A systematic search for downstream mediators of tumor suppressor function of $\mathrm{p} 53$ reveals a major role of BTG2 in suppression of Ras-induced transformation. Genes Dev 20: 236-252, 2006.

30. Rouault JP, Falette N, Guehenneux F, et al: Identification of BTG2, an antiproliferative p53-dependent component of the DNA damage cellular response pathway. Nat Genet 14: 482-486, 1996.

31. Cortes U, Moyret-Lalle C, Falette N, et al: BTG gene expression in the p53-dependent and -independent cellular response to DNA damage. Mol Carcinog 27: 57-64, 2000.

32. Guardavaccaro D, Corrente G, Covone F, et al: Arrest of G (1)-S progression by the p53-inducible gene PC 3 is Rb dependent and relies on the inhibition of cyclin D1 transcription. Mol Cell Biol 20: 1797-1815, 2000.

33. Lim RW, Varnum BC, O'Brien TG and Herschman HR: Induction of tumor promotor-inducible genes in murine 3T3 cell lines and tetradecanoyl phorbol acetate-nonproliferative $3 \mathrm{~T} 3$ variants can occur through protein kinase $\mathrm{C}$-dependent and -independent pathways. Mol Cell Biol 9: 1790-1793, 1989.

34. Ficazzola MA, Fraiman M, Gitlin J, et al: Antiproliferative $B$ cell translocation gene 2 protein is down-regulated posttranscriptionally as an early event in prostate carcinogenesis. Carcinogenesis 22: 1271-1279, 2001.

35. Struckmann K, Schraml P, Simon R, et al: Impaired expression of the cell cycle regulator BTG2 is common in clear cell renal cell carcinoma. Cancer Res 64: 1632-1638, 2004.

36. Kawakubo H, Brachtel E, Hayashida T, et al: Loss of B-cell translocation gene-2 in estrogen receptor-positive breast carcinoma is associated with tumor grade and overexpression of cyclin D1 protein. Cancer Res 66: 7075-7082, 2006. 